\title{
The Value of Brugada Algorithm in the Differential Diagnosis of Broad QRS Complex Tachycardia: A Meta-Analysis
}

\author{
Qiaoying Chai ${ }^{1}$, Gang Liu ${ }^{2}$, HaiJun Zhang ${ }^{1}$ and Mingqi Zheng ${ }^{2}$ \\ ${ }^{1}$ Department of Cardiology, First Hospital of HanDan, HanDan, HeBei, P.R China \\ ${ }^{2}$ Department of Cardiology, First Affiliated Hospital of HeBei Medical University, HeBei, P.R China
}

"Corresponding author: Gang Liu, Department of Cardiology, First Affiliated Hospital of HeBei Medical University, HeBei, P.R China, Tel: 8618633889897; E-mail: zhouwei2009027@126.com

Received: March 17, 2018; Accepted: March 28, 2018; Published: March 30, 2018

Copyright: (c) 2018 Chai Q, et al. This is an open-access article distributed under the terms of the creative commons attribution license, which permits unrestricted use, distribution, and reproduction in any medium, provided the original author and source are credited.

\begin{abstract}
Background: The diagnosis precision of Brugada algorithm in discriminating between supraventricular tachycardia and ventricular tachycardia remains controversial. We aim to evaluate the diagnostic value of Brugada algorithm in broad complex tachycardia arrhythmia.
\end{abstract}

Methods: Eligible studies were identified through searching PubMed, Embase, Web of Science databases, Wan Fang data resource, China National Knowledge Infrastructure prior to 5st January 2018. Studies were assessed for quality using the Quality Assessment for Studies of Diagnostic Accuracy, 2nd version (QUADAS-2). Pooled sensitivity and pooled specificity with $95 \%$ confidence interval $(\mathrm{Cl})$ were calculated using random-effects models. Summary receiver operator characteristic (SROC) curves and the area under curve (AUC) were performed.

Results: A total of 13 studies including 1918 electrocardiogram (ECG) were enrolled in this meta-analysis. The pooled sensitivity and specificity were $0.92(95 \% \mathrm{Cl}: 0.88-0.95)$, and $0.71(95 \% \mathrm{Cl}: 0.57-0.82)$, respectively. The positive likelihood ratio was $3(95 \% \mathrm{Cl}, 2.0-5.0)$ and the negative likelihood ratio was $0.11(95 \% \mathrm{Cl}, 0.06-0.19)$. The summary Diagnostic Odds Ratio (DOR) was 29 (95\% Cl: 12-72). The AUC was 0.95 . Meta-regression analysis showed that sample size, study type and region were not the major sources of heterogeneity, the publication date may account for heterogeneity.

Conclusion: Brugada algorithm has excellent pooled sensitivity and moderate pooled specificity for discriminating SVT from VT. It deserves to be widely used as clinical diagnostic methods.

Keywords: Broad complex tachycardia; Ventricular tachycardia; Supraventricular tachycardia; Brugada algorithm; Meta-analysis

Abbreviations: VT: Ventricular Tachycardia; SVT: Supraventricular Tachycardia; BCT: Broad Complex Tachycardia; SVT-A: Supraventricular Tachycardia with Aberrant Intra-Ventricular Conduction; ECG: Electrocardiography

\section{Introduction}

Broad QRS complex tachycardia (BCT) which defined as a rhythm with a rate $>100 / \mathrm{min}$ and a QRS duration $>120 \mathrm{~ms}$ is a common arrhythmia and often presents a diagnostic dilemma for the physician. Many literatures show that ventricular tachycardia (VT) and supraventricular tachycardia with aberrant intra- ventricular conduction (SVT-A) account for approximately $80 \%$ and $15 \%-20 \%$ of $\mathrm{BCT}$ respectively [1-6]. Other rare reason of BCT, such as Pre-excited tachycardia (PXT) with anterograde conduction through an accessory pathway, supraventricular with drug- and electrolyte-induced abnormal intra-ventricular conduction, ventricular paced rhythm, or electrocardiography (ECG) artifact, account for only a small minority (1\%-5\%). Therefore, the clinically relevant problem in the differential diagnosis of BCTs is to distinguish SVT from VT. The ECG remains the cornerstone of distinguishing SVT from VT. Accurate and immediate differentiation of VT from SVT with aberrancy on surface electrocardiography (ECG) holds significant implications in terms of prognosis and acute and chronic arrhythmia therapeutics.

Although the accurate diagnosis of many existed traditional ECG standards which applied to distinguish SVT from VT are now possible in about $90 \%$ of BCTs $[4,7,8]$. Many of these criteria are not useful in an urgent setting, owing to the complicated and not consistently present. In 1991, Brugada et al. [9] published a study using relatively simple, four-step, decision tree-like algorithm to differentiate between BCTs due to VT and SVT. They reported that this algorithm had a sensitivity (98.7\%) and specificity (96.5\%) remarkable superior to the existed traditional criteria. However, these results have not yet been reproduced independently by other authors who reported a lower sensitivity and specificity $[7,10]$. The accuracy diagnosis of Brugada algorithm in distinguishing BCTs due to SVT or due to VT remains controversial. We therefore performed a meta-analysis of studies evaluating Brugada algorithm in discriminating SVT from VT to establish a more precise estimate of diagnostic accuracy.

\section{Methods}

\section{Article search strategy}

PubMed, Embase, Web of Science databases, Wan Fang data resource, China National Knowledge Infrastructure were searched. The 
Page 2 of 7

following terms were used as keywords: "wide QRS complex tachycardia or broad QRS complex tachycardia", "ventricular tachycardia" or "supraventricular tachycardia" or "differential or distinguish" or "diagnosis" or "Brugada algorithm". The references of identified articles and review articles were also reviewed to involve more relevant studies. The search was performed up to 5th January 2018. We specifically implemented the PubMed search strategy using the terms listed in Table 1.

\begin{tabular}{|l|l|}
\hline Retrieving order & Method \\
\hline$\# 1$ & $\begin{array}{l}\text { (wide QRS complex tachycardia) OR broad QRS } \\
\text { complex (Title/Abstract) }\end{array}$ \\
\hline$\# 2$ & $\begin{array}{l}\text { (ventricular tachycardia AND supraventricular } \\
\text { tachycardia (Transliterated Title) }\end{array}$ \\
\hline$\# 3$ & $\# 1$ OR \#2 \\
\hline$\# 4$ & $\# 3$ AND algorithms (Title/Abstract) \\
\hline$\# 5$ & $\# 3$ AND diagnosis (Title/Abstract) \\
\hline$\# 6$ & $\# 3$ AND Brugada (Title/Abstract) \\
\hline$\# 7$ & $\# 3$ AND aVR lead \\
\hline$\# 8$ & $\# 3$ AND Vereckei (Title/Abstract) \\
\hline$\# 9$ & $\# 3$ OR \#4 OR \#5 OR \#6 OR \#7 OR \#8 \\
\hline
\end{tabular}

Table 1: Pubmed search strategy for meta-analysis.

\section{Study eligibility and quality assessment}

Two observers independently evaluated all articles for relevance using the search strategy at the title, abstract and full-text levels. Disagreements were resolved by a third observer. Studies were included if (1) they attempted to distinguish the ventricular tachycardia (VT) or supraventricular tachycardia with aberrant conduction 9SVT-AC) of broad QRS complex tachycardia by Brugada algorithm; (2) ECGs demonstrate that the broad QRS complex tachycardia is very regular; (3) they were published as full-text articles. The exclusion criteria were as follows: (1) Irregular broad ORS complex tachycardia. (2) Studies that exclusively evaluated the value of the Brugada algorithm in identifying pre-excited tachycardia from broad QRS complex tachycardia. (3) Studies in which specially included idiopathic ventricular tachycardia.

\section{Quality of study reports}

The quality of included studies was assessed according to the quality assessment of diagnostic accuracy studies 2 (QUADAS-2) tool. AQUADAS-2 is used in systematic reviews to evaluate the risk of bias and applicability of diagnostic accuracy studies, and consists of four key domains: patient selection, index test, reference standard, and flow and timing. The assessment for risk of bias was made by every domain and the first three are also evaluated for applicability. Signaling questions were included to assist in judgments about the risk of bias [11]. If the answers to all signaling questions for a domain were "yes," the risk of bias is considered as "low;" when the answers were "no," the "high" risk of bias is judged. The unclear bias should only be used if insufficient information was supplied [11]. Applicability was decided as low, high, or unclear by the similar criteria.

\section{Statistical analysis}

Accuracy estimates: Accuracy data-true positive, false positive, true negative, and false negative-were extracted from each study to calculate estimates of pooled sensitivity and specificity weighted based on the study population size. STATA 13.0 was used to perform Metaanalyses. Sensitivity, specificity, positive likelihood ratio (PLR), negative likelihood ratio (NLR), and diagnostic odds ratio (DOR), forest plots and summary receiver operating characteristic (SROC) curves were analyzed with the 'Midas' module for STATA 13.0, based on the random model effect. Quality of studies was assessed with RevMan 5.2. A well-performing diagnostic precision has an AUC close to 1 , on the contrary an AUC close to 0.5 illustrated a poor performance.

Heterogeneity: Heterogeneity was evaluated by the Cochrane Q test and $\mathrm{I} 2$ test. $\mathrm{P}<0.1$ and $\mathrm{I} 2>50 \%$ indicated that included studies were considered to be with heterogeneity. Subgroup analysis and metaregression were applied to identify the sources of heterogeneity across studies.

Publication bias: Publication bias was evaluated by Deek's funnel asymmetry plot test, and $\mathrm{P}<0.1$ the studies was regarded as existing a significant publication bias statistically.

\section{Results}

\section{Search and selection of the studies}

All total of 427 potentially relevant citations were identified from all searches. 84 articles were excluded because of review literature. Of 343 articles, 265 articles were excluded based on the titles and abstracts. The remaining 78 articles were viewed through full text. Finally, a total of 13 eligible articles satisfying the inclusion criteria were included in the meta-analysis. The detailed procedure of studies searches, and selection was showed in Figure 1.

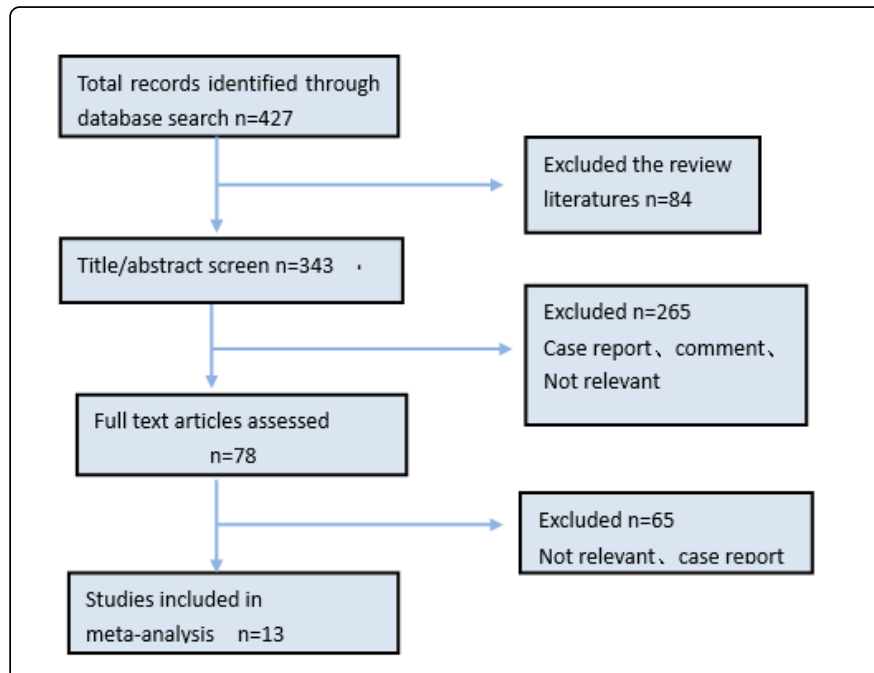

Figure 1: The flow chart of study selection and search result.

\section{Characteristics of included studies}

Clinical characteristics of all the included studies are summarized in Table 2 [12-23]. BCT was defined as a rhythm greater than $100 \mathrm{bpm}$ 
with a QRS longer than $120 \mathrm{~ms}$. Ventricular tachycardia (VT) was defined as a tachycardia that was maintained without the need for structures above the His bundle. The correct diagnosis of the included studies was made on the basis of an electrophysiology study as a golden standard. All Electrocardiograms of BCT were analyzed according to the criterion of Brugada algorithms step by step by the physicians who were blinded regarding the clinical data and the previously established BCT diagnosis. A total of 13 studies comprising 2684 BCTs were incorporated in this analysis, which included 1918 VTs and 769 SVTs with aberrant conduction with proven electrophysiological mechanism. 28.65\% of BCTs were SVTs. Of the 13 studies, 8 studies were prospective, the remainder studies were retrospective. The results of this assessment are given in the "risk of bias summary" in Figure 2.

\section{Diagnostic accuracy of Brugada algorithm}

The pooled sensitivity of Brugada algorithms for diagnosis of BCT was $0.92(95 \%$ CI $0.88-0.95 ; \mathrm{Q}=73.95, \mathrm{P} 0.01 ; 2=83.77 \%)$ and the pooled specificity was 0.71 (95\% CI $0.57-0.82 ; \mathrm{Q}=131.47, \mathrm{P} 0.01 ; 2$ $=90.87 \%$ ), respectively (Figure. 3 ). The positive likelihood ratio was 3 (95\% CI, 2.0-5.0) and the negative likelihood ratio was 0.11 (95\% CI, $0.06-0.19)$.

The summary Diagnostic Odds Ratio (DOR) was 29 (95\% CI: 1272) (Table 3). The SROC curve indicated a relationship between sensitivity and specificity. The area under the curve (AUC) for Brugada algorithm was 0.95 (Figure 4), indicating the highly discriminatory ability of Brugada algorithm.

\section{Publication bias}

We evaluated the publications bias of the studies by Deek's funnel plot. The plot appeared approximately symmetrical, as shown in Figure 5 . The linear regression of log odds ratios on inverse root of effective sample sizes showed that the Bias value was $1.47(\mathrm{P}=0.17)$, which indicated that there was no evidence of publications bias in these included studies (Figure 5).

\section{Heterogeneity assessing and subgroup analysis}

Significant heterogeneity was observed in sensitivity and specificity $(\mathrm{P}<0.01 ; \mathrm{I} 2=83.77 \%$ and $\mathrm{P}<0.01 ; 2=90.87 \%)$ among these 13 studies. Subgroup analysis was performed to identify possible reasons for the heterogeneity. We conducted subgroup analyses based on study sample size, study type, study population and publication date of the study. There were nine studies with sample size greater than 100 ECGs which displayed a pooled sensitivity of 0.93 (95\% CI: 0.87-0.96), specificity of 0.74 (95\% CI: $0.57-0.86)$ and DOR of 37 (95\%CI: 11-130), respectively. The remaining studies with samples less than 100 ECGs, the pooled sensitivity, specificity and DOR were 0.90 (95\% CI: $0.83-$ 0.94), 0.64 (95\% CI: 0.42-0.82) and 16 (95\% CI: 7-35), respectively. In eight prospective studies, the pooled sensitivity, specificity and DOR were 0.93 (95\% CI: 0.88-0.96), 0.80 (95\% CI: 0.65-0.90) and 56(95\% CI: 15-201), respectively.

Five studies were retrospective, in which the pooled sensitivity, specificity and DOR were 0.86 (95\% CI: 0.85-0.91), 0.53 (95\% CI: 0.47-0.61) and 10(95\% CI: 6-16), respectively. Six studies were conducted in Chinese patients, in which the pooled sensitivity, specificity and DOR were 0.91 (95\% CI: 0.83-0.95), 0.77 (95\% CI:
0.61-0.87) and 33 (95\% CI: 10-103), respectively. The remainder seven studies, conducted in patients from the western countries, showed a pooled sensitivity of 0.93 (95\% CI: $0.87-0.96)$, specificity of 0.67 (95\% CI: $0.46-0.83)$ and DOR of 26 (95\% CI: 7-98), respectively.

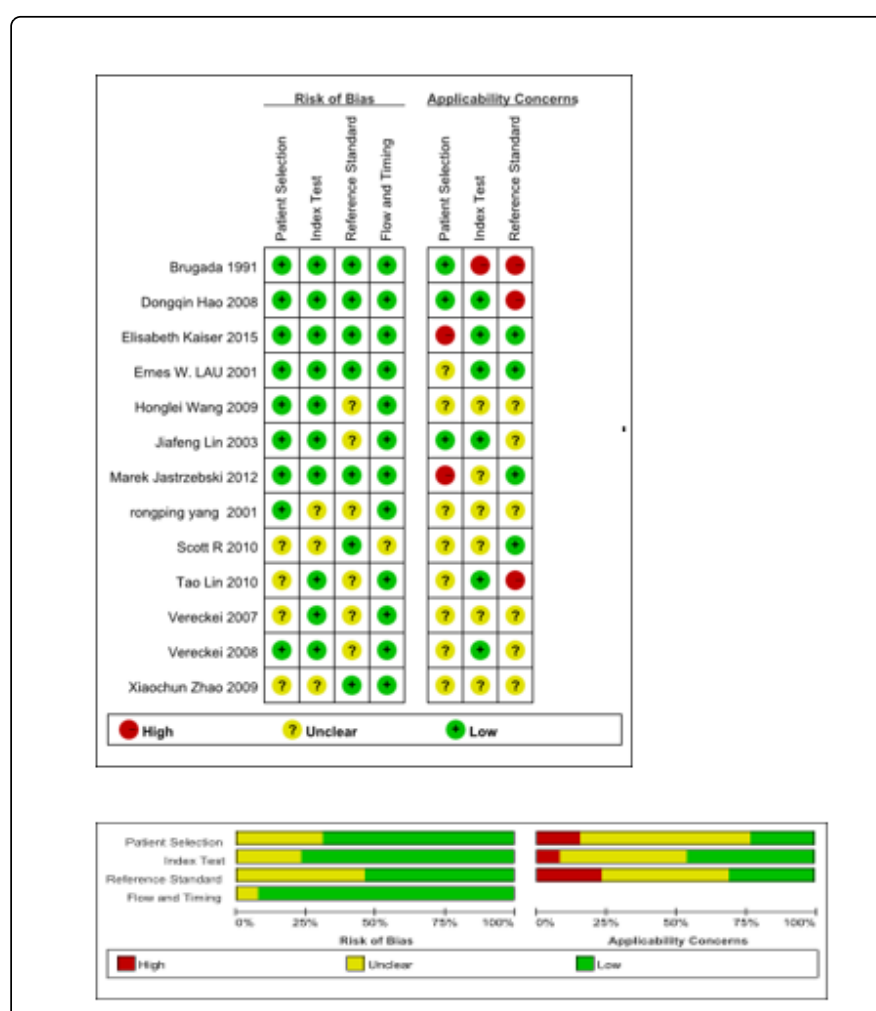

Figure 2: Risk of bias summary.

The subgroup of studies after 2005 included nine studies, whose sensitivity, specificity and DOR were 0.89 (95\% CI: 0.86-0.91), 0.64 (95\% CI: 0.52-0.75) and 14(95\% CI: 9-22), respectively. The remainder studies were prior to 2005 , it wasn't suitable to be analyzed by the STATA.

For meta-regression analysis, we found that these pre-identified confounding covariates, like sample size, study types and region may cause heterogeneity. But these factors were not the major sources of heterogeneity (Figure 6). Meta-regression shows that the publication date would account for the majority of heterogeneity as shown in Figure 7.

In our meta-analysis, there were only 13 studies meeting the requirement with great range of time (from 1991 to 2015) and more than half of these studies were published after 2005 (most of all included studies gathered at 2005 to 2015) would cause the heterogeneity. Additionally, the ECG type and the proportion of every type in included studies were remarkable different, which may be another reason of the heterogeneity. Furthermore, the basic diseases and the situation of heart structure of the patients were not described distinctly in included studies, these differences can also cause the heterogeneity. 
Citation: Chai Q, Liu G, Zhang H, Zheng M (2018) The Value of Brugada Algorithm in the Differential Diagnosis of Broad QRS Complex Tachycardia: A Meta-Analysis. J Cardiovasc Dis Diagn 6: 314. doi:10.4172/2329-9517.1000314

Page 4 of 7

\begin{tabular}{|c|c|c|c|c|c|c|c|c|c|c|}
\hline Study & Year & $\mathbf{N}$ & Age & VT & SVT & PXT & TP & FP & FN & TN \\
\hline $\begin{array}{l}\text { Rong Ping Yang et } \\
\text { al. [12] }\end{array}$ & 2001 & 150 & $50 \pm 2.4$ & 115 & 35 & 8 & 112 & 3 & 2 & 33 \\
\hline $\begin{array}{l}\text { Dong qin Hao et al. } \\
\text { [13] }\end{array}$ & 2008 & 82 & $39.4 \pm 12.5$ & 63 & 19 & 3 & 58 & 6 & 5 & 13 \\
\hline Jiafeng Lin et al. [14] & 2003 & 101 & $50.8 \pm 10.9$ & 58 & 43 & 5 & 54 & 9 & 4 & 34 \\
\hline $\begin{array}{l}\text { Honglei Wang et al. } \\
\text { [15] }\end{array}$ & 2009 & 82 & $37.5 \pm 10.6$ & 57 & 25 & 5 & 46 & 3 & 11 & 22 \\
\hline $\begin{array}{l}\text { Xiaochun Zhao et al. } \\
\text { [16] }\end{array}$ & 2009 & 78 & $43.1 \pm 19.9$ & 66 & 12 & unclear & 60 & 8 & 6 & 4 \\
\hline Tao Lin et al. [17] & 2010 & 113 & 42 & 82 & 31 & unclear & 63 & 8 & 19 & 23 \\
\hline Vereckei et al. [18] & 2007 & 453 & unclear & 348 & 105 & 17 & 307 & 28 & 41 & 77 \\
\hline $\begin{array}{l}\text { Jastrzebski et al. } \\
\text { [19] }\end{array}$ & 2012 & 260 & unclear & 159 & 101 & 23 & 142 & 51 & 17 & 60 \\
\hline Elisabeth et al. [20] & 2015 & 153 & unclear & 111 & 42 & unclear & 100 & 26 & 11 & 16 \\
\hline Vereckei et al. [21] & 2008 & 482 & unclear & 370 & 112 & 20 & 330 & 30 & 40 & 82 \\
\hline $\begin{array}{l}\text { Ernest W Lau et al. } \\
\text { [22] }\end{array}$ & 2002 & 111 & unclear & 77 & 34 & unclear & 73 & 19 & 4 & 15 \\
\hline Brugada et al. [9] & 1991 & 554 & unclear & 384 & 170 & unclear & 379 & 6 & 5 & 164 \\
\hline $\begin{array}{l}\text { Ceresnak SR et al. } \\
\text { [23] }\end{array}$ & 2010 & 65 & $13.5 \pm 5.1$ & 25 & 40 & unclear & 24 & 19 & 1 & 21 \\
\hline
\end{tabular}

SVT: Supraventricular Tachycardia; VT: Ventricular Tachycardia; FN: False Negative; FP: False Positive; TN: True Negative; TP: True Positive; SEN: Sensitivity; SPE: Specificity; PPN: Predictive Positive Value; NPV: Predictive Negative Value

Table 2: Characteristics of included studies.
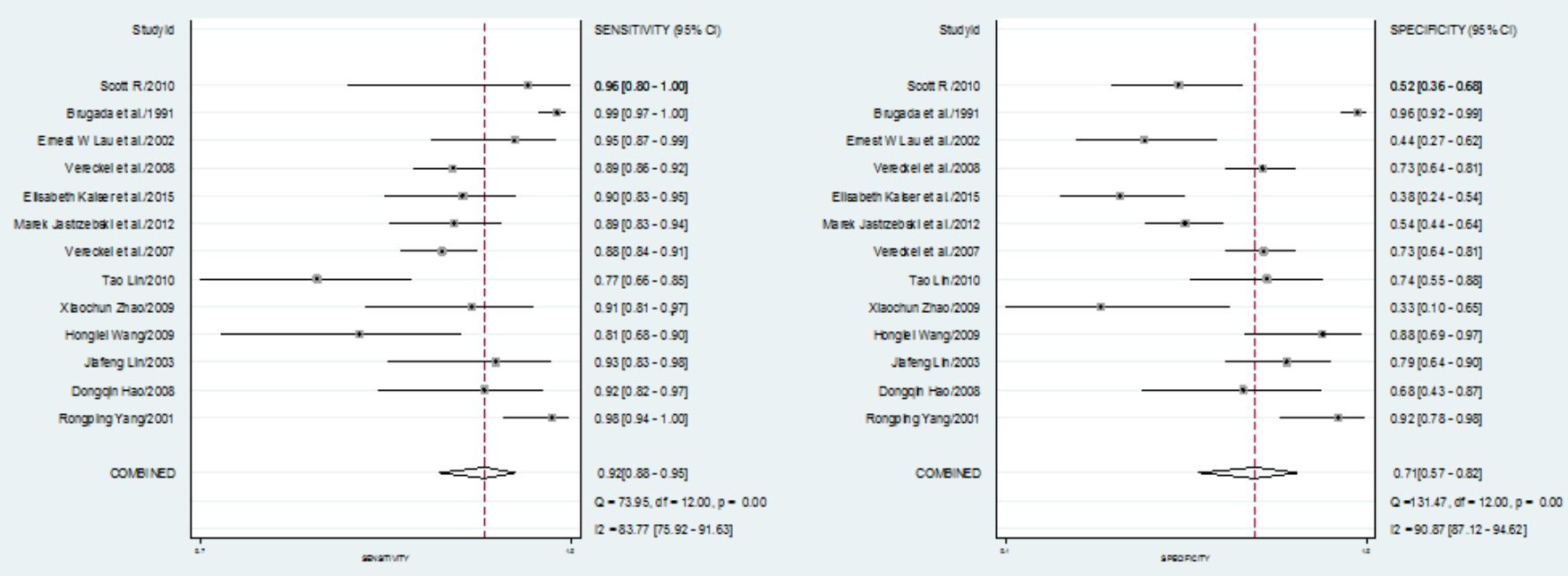

Figure 3: Forest plot of sensitivity and specificity estimates for Brugada algorithm in distinguish of BCT. 


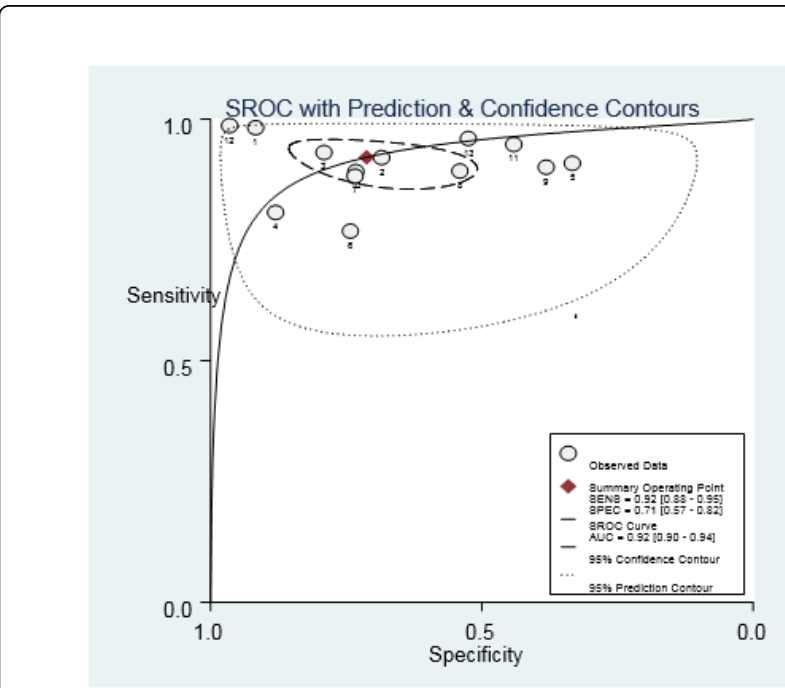

Figure 4: Summary of receiver operator characteristic curve(SROC) for Brugada algorithm in differentiating BCT. AUC: area under the curve.

\begin{tabular}{|l|l|l|}
\hline Parameter & Estimate & $\mathbf{9 5 \%} \mathrm{Cl}$ \\
\hline Sensitivity & 0.92 & $0.88-0.95$ \\
\hline Specificity & 0.71 & $0.57-0.82$ \\
\hline Positive Likelihood Ratio & 3.2 & $2.0-5.0$ \\
\hline Negative Likelihood Ratio & 0.11 & $0.06-0.19$ \\
\hline Diagnostic Odds Ratio & 29 & $12-72$ \\
\hline
\end{tabular}

Table 3: The diagnostic performance of Brugada algorithm.

\section{Discussion}

Traditional criteria, for instance, atria-ventricular (AV) dissociation, QRS greater than $0.14 \mathrm{~ms}$ with right bundle branch block (RBBB), QRS greater than $0.16 \mathrm{~ms}$ with left bundle branch block (LBBB), "positive" concordance in V leads, QRS axis between -90 and -180, LBBB with right axis deviation, and several other morphologies can be conducive to determine VT [6]. Even though these criteria were helpful in the differential diagnosis, mistakes are nevertheless frequently made, particularly in the emergency setting [2,24-27]. Not infrequently, these mistakes have led to wrong therapeutic decisions and therapies which based on a wrong diagnosis may have led to adverse clinical consequences, even went so far as to life-threatening or almost lifethreatening outcomes. Several studies have discussed the limitations of the traditional ECG criteria in the differential diagnosis of a tachycardia with a broad QRS complex. The sensitivity and specificity of the traditional criteria are not optimal. Correct diagnosis is the vital of clinical management, in an effort to help to discriminate between VT and SVT-A, Brugada et al. established a four-step algorithm to facilitate the physician in making the correct diagnosis of BCT due to VT or due to SVT with aberrancy (Figure 8). In the initial survey conducted by Brugada et al. the impressive sensitivity and specificity of the Brugada algorithm were 0.987 and 0.965 , respectively. The simplified step-by-step approach became a welcome accepted method for physician to differentiate this broad complex arrhythmia.

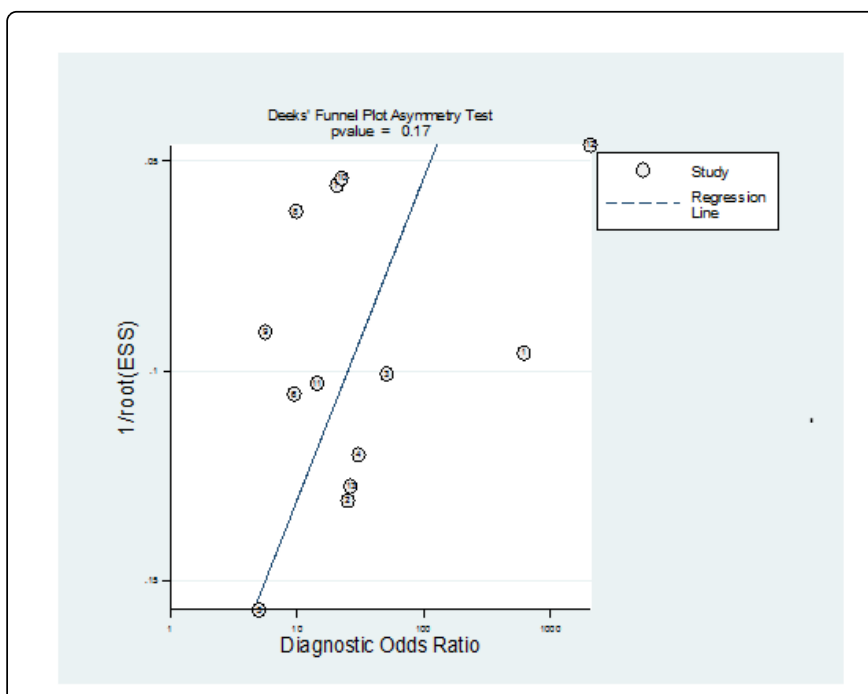

Figure 5: The Deek's funnel asymmetry plot test for evaluation of potential publication bias for Brugada algorithm in the diagnosis of BCT.

In our meta-analyses, we summarized 13 studies involving 2684 BCTs which 1918 ECG was VTs and 769 ECG was SVTs with aberrant conduction. Among regular BCTs, VT is the most prevalent, between $70 \%$ and $75 \%$, as shown in previous studies $[9,21,28,29]$. In Our metaanalysis showed the pooled prevalence of VT was $71.46 \%$, similar to the previous studies. The pooled sensitivity and AUC of Brugada algorithms were more satisfactory (0.92 and 0.95 respectively), while the pooled specificity was moderate $(0.71)$. It suggests that Brugada algorithm would be a useful tool in making the diagnosis of VT, but it may misdiagnose SVT as VT. This misdiagnosis may be unavoidable partly because that no electrocardiographic diagnostic standard with sufficient sensitivity and specificity simultaneously. The performance of a diagnosis method is estimated by the Sensitivity and specificity, but they are often infeasible to secure a high value for both as they are inversely related [30].

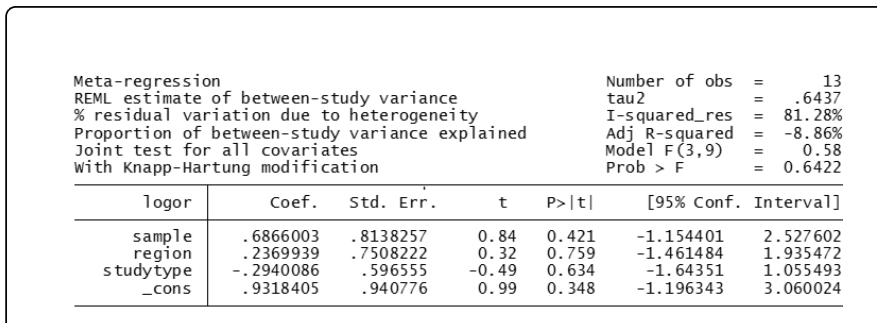

Figure 6: The meta-regression analysis for possible resources of heterogeneity among included studies.

Decision theory analysis shows that the validity of a diagnostic method in practice depends asymmetrically on sensitivity and specificity as well as their interaction with prevalence [31,32]: if prevalence is low, a high specificity is priority to a high sensitivity, oppositely if prevalence is high, a high sensitivity is more pivotal than a high specificity. Based on this theory, with prevalence of 0.70 for VT 
Page 6 of 7

and of 0.30 for SVT in broad complex tachycardia, a method with excellent sensitivity and moderate specificity is acceptable and legitimate to be used as a differential diagnostic criterion of BCT. In clinical practice, VT is misdiagnosed as SVT often with disastrous results or fatal for the patient, so it is better that SVT was misdiagnosed as VT. In our meta-analysis, the pooled sensitivity and pooled specificity of the Brugada algorithm was 0.92 and 0.71 respectively. Therefore, the Brugada algorithm is applicable theoretically and practically.

\begin{tabular}{|c|c|c|c|c|c|c|}
\hline \multicolumn{5}{|c|}{$\begin{array}{l}\text { Meta-regression } \\
\text { REML estimate of between-study variance } \\
\% \text { residual variation due to heterogeneity } \\
\text { Proportion of between-study variance explained } \\
\text { With Knapp-Hartung modification }\end{array}$} & \multirow{2}{*}{$\begin{array}{l}\text { Number of obs } \\
\text { tau2 } \\
\text { I-squared_res } \\
\text { Adj R-squared } \\
{[95 \% \text { Conf. }}\end{array}$} & \multirow{2}{*}{$\begin{array}{l}=13 \\
=.09065 \\
=58.07 \% \\
=84.67 \% \\
\\
\end{array}$} \\
\hline logor & coef. & Std. Err. & $t$ & $P>|t|$ & & \\
\hline $\begin{array}{c}\text { publicatio a } \\
\text { _cons }\end{array}$ & $\begin{array}{l}-.116263 \\
234.6047\end{array}$ & $\begin{array}{r}.024912 \\
50.01985\end{array}$ & $\begin{array}{r}-4.67 \\
4.69\end{array}$ & $\begin{array}{l}0.001 \\
0.001\end{array}$ & $\begin{array}{r}-1710938 \\
124.5118\end{array}$ & $\begin{array}{r}-.0614321 \\
344.6977\end{array}$ \\
\hline
\end{tabular}

Figure 7: The meta-regression analysis of the publication date for possible resources of heterogeneity among included studies.

It is surprising that the pooled sensitivity and pooled specificity of the Brugada algorithm observed in our meta-analysis is significant different from the original study conducted by Brugada et al which the sensitivity and specificity of the Brugada algorithm were 0.987 and 0.965 , respectively. The major discordance was specificity: 0.71 in our study vs. 0.965 in the original study. The interpretation of the significant discrepancy is that the observers in these studies were relatively inexperienced in using the Brugada algorithm compared to those in the original study by Brugada et al. Jennifer $\mathrm{L}$ et al. conducted study showed that to diagnose a same set of broad complex tachycardia electrocardiograph, the sensitivity and the specificity of the Brugada algorithm is different between the emergency physician and cardiologists. Furthermore, neither the emergency physicians nor the cardiologists were able to obtain a sensitivity or specificity as high as the original study [33]. This lack of reproducibility was also reported by others $[10,12-19,23,29]$. Alternatively, it is possible that the BCT types and the proportion of every type in our included studies (except Brugada et al. study) differed from those in the original study; the ECG types in the original study may more suited to the Brugada algorithm than other studies. Idiopathic VTs which their QRS-complex morphology can resemble QRS morphology in some aspects might be difficult to discriminate from SVTs, as they also occur in a healthy heart and some rapidly engage the His-Purkinje network.

Therefore, it must be beer in mind that in most studies that patients with pre-existing bundle branch blocks, pre-excitation, idiopathic VTs, and on anti-arrhythmic drugs were either excluded or underrepresented, or the studies did not show data regarding inclusions, exclusions, or the proportion of such patients in the study. Intentional exclusion or fortuitous underrepresentation of 'difficult' BCT tracings (e g. organic bundle branch blocks, SVTs in heart failure patients, pre-excited tachycardia, and idiopathic VTs) can enhance the performance of an algorithm or differentiation criterion. The second step of the Brugada algorithm that the presence of an RS interval100 $\mathrm{ms}$ in any of the pre-cordial lead is very good at differentiating a clearcut functional aberration from a myocardial scar-related VT, it is not so good when encounter organic left bundle branch block QRS morphology, particularly, in a patient with heart failure, an idiopathic VT or SVP with pre-excitation [34]. It may be the major factor that lowered the SP of the Brugada algorithm.

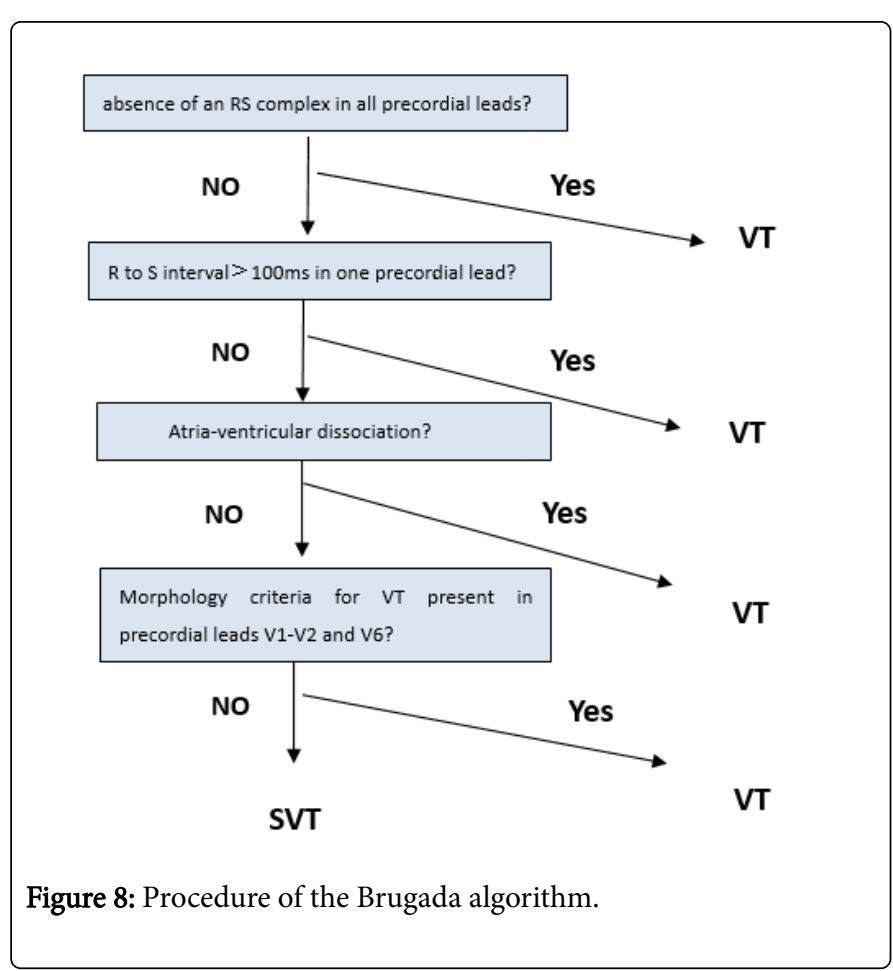

The limitation of our meta-analysis is that our meta-analysis existed high heterogeneity and the meta-regression analyses shows that the publication date may lead to the heterogeneity among studies. The possible reason was that in our meta-analysis, included studies were not sufficient and most of the included studies was published after 2005 compared to before 2005, this contribution of the publication date may cause the heterogeneity. Additionally, the ECG type and the proportion of every type in included studies were remarkably different, which may be another reason of the heterogeneity. Furthermore, the basic diseases and the percentage of every disease of the patients weren't distinctly described, these differences can also cause the heterogeneity. Another limitation of our meta-analysis is that we just valued the diagnostic precision of Brugada algorithm in discriminating SVT from VT but failed to deeply discuss the diagnosis precision of every step of the Brugada algorithm.

\section{Conclusion}

In conclusion, this meta-analysis indicated that Brugada algorithm could be a useful tool with excellent pooled sensitivity and moderate specificity for discriminating SVT from VT. Considered as the most economic, convenient, safe and rapid method, it deserved to be widely used as complementary to other diagnostic techniques.

\section{References}

1. Wellens HJ, Bar FW, Lie KI (1978) The value of the electrocardiogram in the differential diagnosis of a tachycardia with a widened QRS complex. Am J Med 64: 27-33.

2. Stewart RB, Bardy GH, Greene HL (1986) Wide complex tachycardia: misdiagnosis and outcome after emergent therapy. Ann Intern Med 104: 766-771.

3. Baerman JM, Morady F, DiCarlo LA Jr, de Buitleir M (1987) Differentiation of ventricular tachycardia from supraventricular tachycardia with aberration: value of the clinical history. Ann Emerg Med 16: 40-43. 
Citation: Chai Q, Liu G, Zhang H, Zheng M (2018) The Value of Brugada Algorithm in the Differential Diagnosis of Broad QRS Complex Tachycardia: A Meta-Analysis. J Cardiovasc Dis Diagn 6: 314. doi:10.4172/2329-9517.1000314

Page 7 of 7

4. Akhtar M, Shenasa M, Jazayeri M, Caceres J, Tchou PJ (1988) Wide QRS complex tachycardia. Reappraisal of a common clinical problem. Ann Intern Med 109: 905-912.

5. Steinman RT, Herrera C, Schuger CD, Lehmann MH (1989) Wide QRS tachycardia in the conscious adult. Ventricular tachycardia is the most frequent cause. JAMA 261: 1013-1016.

6. Wrenn K (1991) Management strategies in wide QRS complex tachycardia. Am J Emerg Med 9: 592-597.

7. Barold SS (1995) Bedside diagnosis of wide QRS tachycardia. Pacing Clin Electrophysiol 18: 2109-2115.

8. Drew BJ, Scheinman MM (1995) ECG criteria to distinguish between aberrantly conducted supraventricular tachycardia and ventricular tachycardia: practical aspects for the immediate care setting. Pacing Clin Electrophysiol 18: 2194-2208.

9. Brugada P, Brugada J, Mont L, Smeets J, Andries EW (1991) A new approach to the differential diagnosis of a regular tachycardia with a wide QRS complex. Circulation 83: 1649-1659.

10. Alberca T, Almendral J, Sanz P, Almazan A, Cantalapiedra JL, et al. (1997) Evaluation of the specificity of morphological electrocardiographic criteria for the differential diagnosis of wide QRS complex tachycardia in patients with intraventricular conduction defects. Circulation 96: 3527-3533.

11. Whiting PF, Rutjes AW, Westwood ME, Mallett S, Deeks JJ, et al. (2011) QUADAS-2: a revised tool for the quality assessment of diagnostic accuracy studies. Ann Intern Med 155: 529-536.

12. Ping R, Yang XW (2001) Experience of new Brugada criteria for analysis of 150 cases of wide QRS complex tachycardia. J Clin Healthcare 4: 83.

13. Dong Qin Hao HL, Zhao YM (2008) Differential diagnosis value of different algorithms in wide QRS complex tachycardia. Clinical Focus 13: 1452-1454.

14. Feng J, Lin XC, Wang Y, Lou HL, Feng FJ (2003) The value on differential diagnosis of brugada's criteria with steurer's criteria for wide qrs complex tachycardia. Chinese journal of cardiac pacing and electrocardiology 17: 279-283.

15. HongLei Wang SX, PingShuan Dong (2009) Comparation of vereckei and brugadacriteria for differential diagnosis of wide qrs wave tachycardia. J Shandong Medi 49: 42-43

16. XiaoChun Zhao YL, Guo JH, Li XB, Zhang P, Zhang HC (2009) Vereckei and Brugada algorithm for differential diagnosis of wide QRS complex tachycardia. J Clin Electrocardiol 18: 260-263.

17. Lin T, Ma YT, Muhu Y, Tang BP, Hou YM, et al. (2011) Value of aVR lead four steps algorithm on differential diagnosis of wide QRS complex tachycardia. Zhonghua xin xue guan bing za zhi 39: 69-72.

18. Vereckei A, Duray G, Szenasi G, Altemose GT, Miller JM (2007) Application of a new algorithm in the differential diagnosis of wide QRS complex tachycardia. Eur Heart J 28: 589-600.
19. Jastrzebski M, Kukla P, Czarnecka D, Kawecka-Jaszcz K (2012) Specificity of the wide QRS complex tachycardia algorithms in recipients of cardiac resynchronization therapy. J Electrocardiol 45: 319-326.

20. Kaiser E, Darrieux FC, Barbosa SA, Grinberg R, Assis-Carmo A, et al. (2015) Differential diagnosis of wide QRS tachycardias: comparison of two electrocardiographic algorithms. Europace 17: 1422-1427.

21. Vereckei A, Duray G, Szenasi G, Altemose GT, Miller JM (2008) New algorithm using only lead aVR for differential diagnosis of wide QRS complex tachycardia. Heart rhythm 5: 89-98.

22. Lau WE, Andre NG (2002) Comparison of the performance of three diagnostic algorithms for regular broad complex tachycardia in Practical Application. PACE 25: 822-827.

23. Ceresnak SR, Liberman L, Avasarala K, Tanel R, Motonaga KS, et al (2010) Are wide complex tachycardia algorithms applicable in children and patients with congenital heart disease? J Electrocardiol 43: 694-700.

24. DiMarco JP, Lerman BB (1985) Role of invasive electrophysiologic studies in the evaluation and treatment of supraventricular tachycardia. Pacing Clin Electrophysiol 8: 132-139.

25. Morady F, Baerman JM, DiCarlo LA Jr, DeBuitleir M, Krol RB, et al. (1985) A prevalent misconception regarding wide-complex tachycardias. JAMA 254: 2790-2792.

26. Morgan DE, Brennan FJ (1985) Diagnostic potential for verapamil in wide QRS complex tachycardia. Am J Cardiol 55: 1428-1429.

27. Kindwall KE, Brown J, Josephson ME (1988) Electrocardiographic criteria for ventricular tachycardia in wide complex left bundle branch block morphology tachycardias. Am J Cardiol 61: 1279-1283.

28. Lau EW, Pathamanathan RK, Ng GA, Cooper J, Skehan JD, et al. (2000) The Bayesian approach improves the electrocardiographic diagnosis of broad complex tachycardia. Pacing Clin Electrophysiol 23: 1519-1526.

29. Lau EW, Ng GA (2001) Comparison of two diagnostic algorithms for regular broad complex tachycardia by decision theory analysis. Pacing Clin Electrophysiol 24: 1118-1125.

30. Altman DG, Bland JM (1994) Diagnostic tests. 1: Sensitivity and specificity. BMJ 308: 1552.

31. Hilden J (2000) Prevalence-free utility-respecting summary indices of diagnostic power do not exist. Stat Med 19: 431-440.

32. Hilden J GP (1996) Regret graphs, diagnostic uncertainty and youdens index. Stat Med 15: 969-986.

33. Isenhour JL, Craig S, Gibbs M, Littmann L, Rose G, et al. (2000) Widecomplex tachycardia: Continued evaluation of diagnostic criteria. Acad Emerg Med 7: 769-773.

34. Jastrzebski M, Moskal P, Kukla P, Fijorek K, Kisiel R, et al. (2017) Specificity of wide QRS complex tachycardia criteria and algorithms in patients with ventricular preexcitation. Ann Noninvasive Electrocardiol e12493. 